\title{
Physiologically Structured Population Models: Towards a General Mathematical Theory
}

Odo Diekmann (O.Diekmann@math.ruu.nl)

Mats Gyllenberg (mats.gyllenberg@helsinki.fi)

Johan A.J. Metz (J.A.J.Metz@biology.leidenuniv.nl)

\section{Approved by}

Ulf Dieckmann

Leader, Evolution and Ecology Program

December 2007 


\section{Contents}

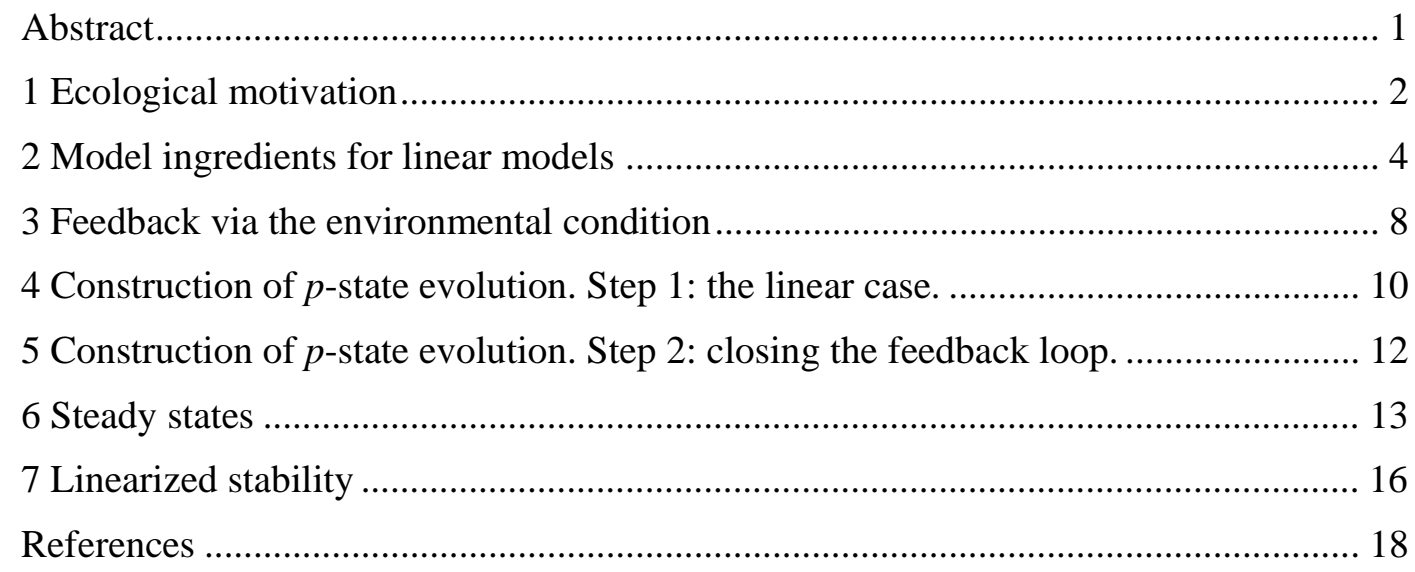




\title{
Physiologically structured population models: Towards a general mathematical theory
}

\author{
O. Diekmann \\ Department of Mathematics, University of Utrecht, \\ P.O. Box 80010, 3580 TA Utrecht, The Netherlands \\ O.Diekmann@math.ruu.nl \\ M. Gyllenberg \\ Rolf Nevanlinna Institute \\ Department of Mathematics and Statistics, \\ FIN-00014 University of Helsinki, Finland \\ mats.gyllenberg@helsinki.fi
}

\section{J.A.J. Metz}

Institute for Evolutionary and Ecological Sciences, Leiden University, Kaiserstraat 63, NL-2311 GP Leiden, The Netherlands and

, IIASA, A-2361 Laxenburg, Austria. metz@rulsfb.leidenuniv.nl

\begin{abstract}
We review the state-of-the-art concerning a mathematical framework for general physiologically structured population models. When individual development is affected by the population density, such models lead to quasilinear equations. We show how to associate a
\end{abstract}


dynamical system (defined on an infinite dimensional state space) to the model and how to determine the steady states. Concerning the principle of linearized stability, we offer a conjecture as well as some preliminary steps towards a proof.

\section{Ecological motivation}

How do phenomena at the population level ( $p$-level) relate to mechanisms at the individual level ( $i$-level)? When investigating the relationship, it is often necessary to distinguish individuals from one another according to certain physiological traits, such as body size and energy reserves. The resulting p-models are called "physiologically structured" (Metz and Diekmann 1986). They combine an $i$-level submodel for "maturation", i.e., change of $i$-state, with submodels for "survival" and "reproduction", which concern changes in the number of individuals. So they are "individual based", in the sense that the submodels apply to processes at the $i$-level. Yet they usually (but not necessarily) employ deterministic bookkeeping at the $p$-level (so they involve an implicit "law of large numbers" argument).

A first aim of this paper is to explain a systematic modelling approach for incorporating interaction. The key idea is to build a nonlinear model in two steps, by explicitly introducing, as step one, the environmental condition via the requirement that individuals are independent from one another (and hence equations are linear) when this condition is prescribed as a function of time. The second step then consists of modelling the feedback law that describes how the environmental condition depends on the current population size and composition.

Let us sketch three examples, while referring to de Roos and Persson (2001, 2002) and de Roos, Persson and McCauley (2003) for more details, additional examples and motivation as well as further references.

If juveniles turn adult (i.e., start reproducing) only upon reaching a certain size, there is a variable maturation delay between being born and reaching adulthood. Since small individuals need less energy for maintenance than large individuals, the juveniles can outcompete their parents by reducing the food level so much that adults starve to death. Thus "cohort cycles" may result, i.e., the population can consist of a cohort of individuals which are all born within a small time window. Once the cohort reaches the adult size it starts reproducing, thus producing the next cohort, but then quickly dies 
from starvation. So here the $p$-phenomenon is the occurrence of cohort cycles (which are indeed observed in fish populations in several lakes (Persson et al. 2000)) and the $i$-mechanism is the combination of a minimal adult size with a food concentration dependent $i$-growth rate.

The second example concerns cannibalistic interaction. Again we take $i$ size as the $i$-state, now since bigger individuals can eat smaller ones, but not vice versa. The $p$-phenomenon is that a population may persist at low renewal rates for adult food, simply since juvenile food becomes indirectly available to adults via cannibalism (the most extreme example is found in some lakes in which a predatory fish, such as pike or perch, occurs but no other fish whatsoever, cf. Persson et al. 2000, 2003). So reproduction becomes similar to farming, gaining a harvest from prior sowing (Getto, Diekmann and de Roos, submitted).

The third example is a bit more complex. It concerns the interplay between competition for food and mortality from predation in a size structured consumer population that is itself prey to an exploited (by humans) predator population, where the predators eat only small prey individuals. The phenomenon of interest is a bistability in the composition of the consumer population with severe consequences for the predators. At low mortality from predation, a large fraction of the consumers pass through the vulnerable size range, leading to a severe competition for food and a very small per capita as well as total reproductive output. The result is a consumer population consisting of stunted adults and few juveniles, a size structure that keeps the predators from (re-)entering the ecosystem. However, if the ecosystem is started up with a high predator density, due to a history in which parameters were different, these predators, by eating most of the young before they grow large, cause the survivors to thrive, with a consequent large total reproductive output. Thus, the predators keep the density of vulnerable prey sufficiently high for the predator population to persist. If exploitation lets the predator population diminish below a certain density, it collapses due to the attendant change in its food population.

Interestingly, a similar phenomenon can occur if the predators preferentially eat the larger sized individuals only. A more detailed analysis by de Roos, Persson and Thieme (2003) shows that the essence of the matter is that in the absence of predators the consumer population is regulated mainly by the rate at which individuals pass through a certain size range, with the predators specialising on a different size range. As noted by de Roos and Persson (2002), a mechanism of this sort may well explain the failure of the 
Northwest Atlantic cod to recover after its collapse from overfishing: After the cod collapsed, the abundance of their main food, capelin, increased, but capelin growth rates decreased and adults became significantly smaller. (See Scheffer et al. (2001) for a general survey on catastrophic collapses.)

A large part of this paper is based on earlier work of ours, viz. (Diekmann et al. 1998, 2001, 2003), which we shall refer to as Part I, Part II, and Part III, respectively. The reader is referred to (Ackleh and Ito, to appear; Calsina and Saldaña, 1997; Cushing, 1998; Tucker and Zimmermann, 1988) for alternative approaches.

\section{Model ingredients for linear models}

Let the $i$-state, which we shall often denote by the symbol $x$, take values in the $i$-state space $\Omega$. Usually $\Omega$ will be a nice subset of $\mathbf{R}^{\mathbf{k}}$ for some $k$. As an example, let $x=\left(\begin{array}{l}a \\ y\end{array}\right)$ with $a$ the age and $y$ the size of an individual. Then $\Omega$ could be the positive quadrant $\{x: a \geq 0, y \geq 0\}$ or some subset of this quadrant.

We denote the environmental condition, either as a function of time or at a particular time, by the symbol $I$. In principle $I$ at a particular time is a function of $x$, since the way individuals experience the world may very well be $i$-state specific. For technical reasons, we restrict our attention to environmental conditions that are fully characterized in terms of finitely many numbers (i.e., $I(t) \in \mathbf{R}^{\mathbf{k}}$ for some $k$ and $x$-dependence is incorporated via fixed weight functions as explained below by way of an example). The technical reasons are twofold. Firstly, this seems a necessary approximation when it comes to numerical solution methods. Secondly, as yet we have not developed any existence and uniqueness theory for the initial value problem in cases in which the environmental condition is $i$-state specific (and to do so one has to surmount substantial technical problems (Kirkilionis and Saldaña, in preparation).

As an example, think of $I=\left(\begin{array}{c}I_{1} \\ I_{2}\end{array}\right)$, with $I_{1}$ the concentration of juvenile food and $I_{2}$ the concentration of adult food. We may then describe the food concentration as experienced by an individual of size $y$ by the linear combination $\phi_{1}(y) I_{1}+\phi_{2}(y) I_{2}$, where $\phi_{1}$ is a decreasing function while $\phi_{2}$ is increasing. Thus we can incorporate that the food preference is $y$-specific 
and gradually changes from juvenile to adult food.

The environmental condition should be chosen such that individuals are independent from one another when $I$ is given as a function of time. The $i$-state should be such that all information about the past of $I$, relevant for predicting future $i$-behaviour, is incorporated in the current value of the $i$ state. Here " $i$-behaviour" first of all refers to contribution to population changes, i.e., to death and reproduction (note that at the $i$-level this may very well amount to specifying probabilities per unit of time), but once the $i$-state has been introduced it also refers to predicting future $i$-states from the current $i$-state (possibly in the form of specifying a probability density).

As a notational convention we adopt that an environmental condition $I$ is defined on a time interval $[0, \ell(I))$. Often we call $I$ an input and $\ell(I)$ the length of the input. For $s \leq \ell(I)$ we then denote by $\rho(s) I$ the restriction of $I$ to the interval $[0, s)$. By defining

$$
(\theta(-s) I)(\tau)=I(\tau+s) \text { for } 0 \leq \tau<\ell(I)-s
$$

we achieve that $\theta(-s) I$ incorporates the information about the restriction of $I$ to $[s, \ell(I))$ but, by shifting back, in the form of a function defined on $[0, \ell(I)-s)$. We write

$$
I=\theta(-s) I \odot \rho(s) I
$$

where the symbol $\odot$ denotes concatenation defined by

$$
(J \odot K)(\tau)= \begin{cases}K(\tau) & 0 \leq \tau<\ell(K) \\ J(\tau-\ell(K)) & \ell(K) \leq \tau<\ell(K)+\ell(J)\end{cases}
$$

A linear structured population model is defined in terms of two ingredients, $u$ and $\Lambda$, which are both functions of $I, x$ and $\omega$, where $\omega$ is a measurable subset of $\Omega$ (which thus implies the requirement that $\Omega$ comes equipped with a $\sigma$-algebra $\Sigma$ ). The interpretation is as follows:

$u_{I}(x, \omega)$ is the probability that, given the input $I$, an individual which has $i$-state $x \in \Omega$ at a certain time, is still alive $\ell(I)$ units of time later and then has $i$-state in $\omega \in \Sigma$;

$\Lambda_{I}(x, \omega)$ is the number of offspring, with state-at-birth in $\omega \in \Sigma$, that an individual is expected to produce when it gets exposed to the input $I$ while starting in $x$, during the total length of the input.

This interpretation requires that certain consistency relations and monotonicity conditions should hold. In order to formulate these we first introduce some 
terminology and notation. We want $u$ and $\Lambda$ to be parametrized positive kernels, where $I$ is the "parameter" and a kernel $k$ is a map from $\Omega \times \Sigma$ into $\mathbf{R}$ which is bounded and measurable with respect to the first variable and countably additive with respect to the second variable. We call a kernel positive if it assumes non-negative values only. The product $k \times l$ of two kernels $k$ and $l$ is the kernel defined by

$$
(k \times l)(x, \omega)=\int_{\Omega} k(\xi, \omega) l(x, d \xi) .
$$

\section{Assumption 2.1}

(i) Chapman-Kolmogorov:

$$
u_{I \odot J}=u_{I} \times u_{J}
$$

(ii) Reproduction-survival-maturation consistency:

$$
\Lambda_{I \odot J}=\Lambda_{J}+\Lambda_{I} \times u_{J}
$$

(iii) $\sigma \mapsto \Lambda_{\rho(\sigma) I}(x, \omega)$ is non-decreasing with limit zero for $\sigma \downarrow 0$ (the monotonicity actually follows from (2.6) and positivity).

(iv) $\sigma \mapsto u_{\rho(\sigma) I}(x, \Omega)$ is non-increasing and

$$
\lim _{\sigma \downarrow 0} u_{\rho(\sigma) I}(x, \omega)=\delta_{x}(\omega) .
$$

(v) In addition we require finite life expectancy: there exists $M<\infty$ such that

$$
\int_{(0, \ell(I))} \sigma u_{\rho(d \sigma) I}(x, \Omega) \leq M
$$

for all $x \in \Omega$ and all $I$.

If maturation is deterministic, the ingredient $u_{I}$ can be put into a particularly simple and useful form. Consider an individual with $i$-state $x$ at a certain time. Let $X_{I}(x)$ be the $i$-state of that individual $\ell(I)$ units of time later, given the input $I$ and let $\mathcal{F}_{I}(x)$ be its survival probability. Then

$$
u_{I}(x, \omega)=\mathcal{F}_{I}(x) \delta_{X_{I}(x)}(\omega) .
$$

Concerning the specification of $\Lambda$, it makes first of all sense to introduce the set $\Omega_{b}$ of possible states-at-birth (cf. Part I, Definition 2.5; the idea is that $\Lambda_{I}(x, \omega)=0$ whenever $\left.\omega \cap \Omega_{b}=\emptyset\right)$. Two situations are of special interest 
- the discrete case: $\Omega_{b}$ is a finite set $\left\{x_{b_{1}}, x_{b_{2}}, \ldots, x_{b_{m}}\right\}$ (with the case $m=1$ being of even stronger special interest)

- the absolutely continuous case: $\Omega_{b}$ is a lower dimensional manifold with a "natural" (Lebesgue) measure $d \xi$ defined on it, and $\Lambda_{I}(x, \cdot)$ is absolutely continuous with respect to that measure. Here the archetypical example is $\Omega_{b}=\left\{(a, x): a=0, x_{\min } \leq x \leq x_{\max }\right\}$ that arises when modeling an age-size structured population.

In the case of a finite $\Omega_{b}$ we put

$$
\Lambda_{I}(x, \omega)=\sum_{j=1}^{m}{ }_{j} L_{I}(x) \delta_{x_{b_{j}}}(\omega)
$$

where ${ }_{j} L_{I}(x)$ is the expected number of children, with $i$-state at birth $x_{b_{j}}$, produced, given the input $I$ and in the period of length $\ell(I)$ of this input, by an individual having $i$-state $x$ at the start of the input. In the case of $\Omega_{b}$ being a lower dimensional manifold we put

$$
\Lambda_{I}(x, \omega)=\int_{\omega \cap \Omega_{b}} \xi L_{I}(x) d \xi,
$$

where ${ }_{\xi} L_{I}(x)$ has an analogous interpretation (but note that now it is a density with respect to $\xi$ : only after integrating with respect to $\xi$ over a subset of $\Omega_{b}$ do we get a number).

The building blocks $X, \mathcal{F}$ and $L$ are, in turn, obtained as solutions of differential equations when the $i$-model is formulated in terms of a maturation rate $g$, a per capita death rate $\mu$ and a per capita (state-at-birth specific) reproduction rate $\beta$. These read

$$
\begin{aligned}
& \left\{\begin{array}{l}
\frac{d}{d t} X_{\rho(t) I}(x)=g\left(X_{\rho(t) I}(x), I(t)\right) \\
X_{\rho(0) I}(x)=x
\end{array}\right. \\
& \left\{\begin{array}{l}
\frac{d}{d t} \mathcal{F}_{\rho(t) I}(x)=-\mu\left(X_{\rho(t) I}(x), I(t)\right) \mathcal{F}_{\rho(t) I}(x) \\
\mathcal{F}_{\rho(0) I}(I)=1
\end{array}\right. \\
& \left\{\begin{array}{l}
\frac{d}{d t} \xi L_{\rho(t) I}(x)=\beta_{\xi}\left(X_{\rho(t) I}(x), I(t)\right) \mathcal{F}_{\rho(t) I}(x) \\
\xi L_{\rho(0) I}(x)=0
\end{array}\right.
\end{aligned}
$$


or, in short hand notation,

$$
\left\{\begin{aligned}
\frac{d X}{d t} & =g(X, I) \\
\frac{d \mathcal{F}}{d t} & =-\mu(X, I) \mathcal{F} \\
\frac{d L}{d t} & =\beta(X, I) \mathcal{F}
\end{aligned}\right.
$$

We conclude that the ingredients $u$ and $\Lambda$ for a linear structured population model can be constructively defined in terms of solutions $X, \mathcal{F}$ and $L$ of ordinary differential equations involving the ingredients $g, \mu$ and $\beta$ which specify the $i$-behaviour in terms of rates as a function of the current $i$-state and the prevailing environmental condition.

When $i$-state development is stochastic, rather than deterministic, one needs to replace (2.10). For instance, if $i$-state corresponds to spatial position and individuals perform Brownian motion, one needs to replace (2.10) by the diffusion equation for the probability density of finding the individual at a position after some time, given $I$. The advantage of "starting" from the ingredients $u$ and $\Lambda$ is that they encompass all such variations.

It is straightforward to check that, under appropriate assumptions on $g, \mu$ and $\beta,(2.7)-(2.8) /(2.9)$ define parametrised positive kernels satisfying Assumption 2.1.

The true modelling consists of a specification of $g, \mu$ and $\beta$, see e.g. (Kooijman, 2000).

\section{Feedback via the environmental condition}

At any time $t$ a population is described by a positive measure $m(t)$ on $\Omega$. Possibly this measure is absolutely continuous (with respect to the Lebesgue measure; again we think of $\Omega$ as a subset of $\mathbf{R}^{\mathbf{k}}$ ). Then there is a density function $n(t, \cdot)$, defined on $\Omega$, such that

$$
m(t)(\omega)=\int_{\omega} n(t, x) d x .
$$

To illustrate the idea of interaction via environmental variables, we consider the situation of competition for food. Let the dynamics of the substrate $S$ be generated by

$$
\frac{d S}{d t}=\frac{1}{\varepsilon}\left(S_{0}-S-S \int_{\Omega} \gamma(x) m(t)(d x)\right),
$$


where $\varepsilon^{-1} \gamma$ is the $i$-state specific per capita consumption rate. So an individual with $i$-state $x$ ingests $\varepsilon^{-1} \gamma(x) S$ units of substrate per unit of time. In energy budget models (Kooijman, 2000) one often assumes that a fraction $1-\kappa(x)$ of the ingested energy is scheduled to growth and maintenance and the remaining fraction $\kappa(x)$ to reproduction. Thus the $\varepsilon^{-1} \gamma(x) S$ enters in the specification of $g$ and $\beta$ (and, in case of starvation, i.e., when maintenance cannot be covered, also $\mu$ ). So the $S$ is (a component of) $I$. Vice versa, the factor $\int_{\Omega} \gamma(x) m(t)(d x)$ corresponds to the environmental condition for the substrate population. It appears that we can couple the substrate and the consumer population via the idea that one constitutes the environmental condition for the other.

If the time scale parameter $\varepsilon$ in (3.2) is very small one can employ the quasi-steady-state approximation for the substrate, i.e., require that the factor within brackets at the right hand side of (3.2) equals zero. This yields

$$
S=\frac{S_{0}}{1+I}
$$

where

$$
I(t)=\int_{\Omega} \gamma(x) m(t)(d x)
$$

One should interpret these two identities as follows. When $I$ is considered as given, as an input, the formula (3.3) specifies what substrate density the individuals of the consumer population experience. And this then in turn determines how the $I$ enters the expressions for $g, \beta$ and, possibly, $\mu$. The identity (3.4), on the other hand, is the feedback law specifying how, in fact, the $I$ at a particular time relates to the extant population at that time. In other words, the combination of (3.3) with rules for how $g, \beta$ and $\mu$ depend on $S$ defines a linear structured population model. But if we add to that the consistency requirement (3.4) we turn the linear model into a nonlinear model in which it is incorporated that individuals interact by competing for a limited resource $S$. Note that the ingredients $g, \mu$ and $\beta$ of the linear model need to be supplemented by the ingredient $\gamma$ in order to define the nonlinear model. One could call $\gamma(x)$ the $i$-state specific contribution to the environmental condition. (The precise interpretation depends on the meaning of (the component of) $I$ ).

Since the environmental condition is chosen such that individuals are, for given $I$, independent of one another, the feedback law (3.4) is necessarily linear. Or, phrased differently, the components of $I$ are linear functionals of the $p$-state. We call (3.4) a pure mass-action feedback law. 
Sometimes the specification of $g, \mu$ and $\beta$ is based on submodels for behavioural processes at a very short time scale, the most well-known example being the Holling type II functional response as derived from a submodel in which predators can be either searching for prey or busy handling prey that has been caught. In such cases the feedback law exhibits a certain hierarchical structure which is described in Part II, Section 6 and which we have called generalized mass action. In this paper we restrict ourselves to the pure mass action case (3.4).

Especially in the modeling phase it is often helpful to close the feedback loop in two steps: first an output is computed, which then is fed back as input via a feedback map. In the example considered above we would write (3.4) as

$$
O(t):=\int_{\Omega} \gamma(x) m(t)(d x) .
$$

Considering $S$ as the true input, we would then write (3.3) as

$$
S=F(O)
$$

where

$$
F(O)=\frac{S_{0}}{1+O}
$$

The advantage is twofold: i) it represents better what is going on biologically, and ii) one can use (3.5) as a definition, with (3.6) as the equation that closes the feedback loop. In contrast (3.3), by combining both steps in one, lacks such a clear interpretation. From a mathematical viewpoint the role of (3.4) is that of an equation only, while the modelling aspect, i.e., the definition of what inputs and outputs amount to observationally, is lost from sight. On the other hand, the drawback of distinguishing between $I$ and $O$ is that an additional variable is introduced which clutters the analysis without playing any useful role. So in the following we use onlu $I$.

\section{Construction of $p$-state evolution. Step 1: the linear case.}

For the sake of exposition we restrict ourselves here to the situation of a fixed state-at-birth $x_{b}$. Given an initial $p$-state $m$, we define the cumulative first 
generation offspring function $B^{1}$ by

$$
B^{1}(t)=\int_{\Omega} L_{\rho(t) I}(x) m(d x) .
$$

The cumulative second generation offspring function $B^{2}$ is next defined by

$$
B^{2}(t)=\int_{0}^{t} L_{\rho(t-\tau) \theta(-\tau) I}\left(x_{b}\right) B^{1}(d \tau),
$$

et cetera (that is, replace in (4.2) $B^{2}$ by $B^{n+1}$ and $B^{1}$ by $B^{n}$ ). The cumulative "all offspring" function

$$
B^{c}=\sum_{n=1}^{\infty} B^{n}
$$

then satisfies the renewal equation

$$
B^{c}(t)=B^{1}(t)+\int_{0}^{t} L_{\rho(t-\tau) \theta(-\tau) I}\left(x_{b}\right) B^{c}(d \tau)
$$

and one can view (4.3) as the generation expansion obtained by solving (4.4) by successive approximation. Note that $B^{c}$ depends on $I$, even though we do not incorporate this in the notation.

If we denote by $T_{I} m$ the $p$-state at time $\ell(I)$, given that the $p$-state at time zero is $m$ and given the time course $I$ of the environmental condition, then

$$
T_{I} m=u_{I} \times m+\int_{0}^{\ell(I)} u_{\theta(-\tau) I}\left(x_{b}, \cdot\right) B^{c}(d \tau)
$$

where

$$
\left(u_{I} \times m\right)(\omega)=\int_{\Omega} u_{I}(x, \omega) m(d x)
$$

describes the survival and maturation of the individuals present at time zero, while the second term takes into account the survival and maturation of all individuals born after time zero. The key result of Part I is that the operators $T_{I}$ form a semigroup, that is, the map $I \mapsto T_{I}$ transforms concatenation (recall(2.2)) into composition of maps:

Theorem 4.1

$$
T_{I}=T_{\theta(-t) I} T_{\rho(t) I}
$$

for any $t \in[0, l(I))$ 
Let us recapitulate. Starting from $g, \mu$ and $\beta$, one constructs $u$ and $L$ (recall (2.8)); if there is only one possible state-at-birth, then $\Lambda$ is completely determined by $L$ ). Given an initial $p$-state $m$ one next constructively defines the solution $B^{c}$ of (4.4) by (4.3). The formula (4.5) then provides a way to calculate, given $I$, the $p$-state after $\ell(I)$ units of time from $u, B^{c}$ and $m$. And Theorem 4.1 justifies our use of the word " $p$-state": our construction yields a dynamical system.

Even though we rightfully refer to Part I for Theorem 4.1, readers who want to see more details are advised to first consult Part II since some of our current notation goes back only to that reference.

\section{Construction of $p$-state evolution. Step 2: closing the feedback loop.}

If we substitute $m(t)=T_{\rho(t) I} m$ into (3.4) we obtain the equation

$$
I(t)=\gamma \times T_{\rho(t) I} m=\int_{\Omega} \gamma(x)\left(T_{\rho(t) I} m\right)(d x)
$$

that $I$ should satisfy in order to have consistency between input and output. We view (5.1) as a fixed point problem for $I$, parametrised by the initial $p$-state $m$.

In Sections 7 and 8 of Part II one finds various assumptions on $u, \Lambda$ and $\gamma$, respectively, $g, \mu, \beta$ and $\gamma$ that guarantee that the right hand side of (5.1) defines a contraction mapping on a suitable function space. Here "suitable" in particular involves a restriction for the length $l$ of the interval on which $I$ is defined. Thus the contraction mapping principle yields a local solution $I=I_{m}$ of (5.1). One next notes (see Diekmann and Getto, to appear, for details) that:

- a fixed point on a smaller interval is a restriction of a fixed point on a larger interval,

$-\theta(-t) I_{m}=I_{T_{\rho(t) I m} m}$, roughly saying that shifted fixed points are the fixed points corresponding to the updated $p$-state,

- uniqueness holds on any interval,

- fixed points can be concatenated to achieve continuation, that is, to obtain solutions on longer time intervals 
to conclude that the local solution can be extended to a maximal solution, which we also denote by $I_{m}$. A key result of Part II is that the definition

$$
S(t, m)=T_{\rho(t) I_{m}} m
$$

yields a semiflow:

\section{Theorem 5.1}

$$
S(t+s, m)=S(t, S(s, m))
$$

(Again we refer to Diekmann \& Getto, to appear, for details and for various results about boundedness and global existence as well as weak*-continuity with respect to time $t$ and initial condition $m$.)

\section{$6 \quad$ Steady states}

The symbol $\bar{I}$ denotes a constant input defined on $[0, \infty)$. (Slightly abusing notation we do not distinguish between the function and the value it takes.) A steady state is a measure $\bar{m}$ on $\Omega$ such that

$$
T_{\rho(t) \bar{I}} \bar{m}=\bar{m}, \quad \forall t \geq 0
$$

where

$$
\bar{I}=\gamma \times \bar{m}=\int_{\Omega} \gamma(x) \bar{m}(d x) .
$$

Since $\tilde{T}(t):=T_{\rho(t) \bar{I}}$ is a semigroup of positive linear operators and $\bar{m}$ has to be positive, (6.1) amounts to the condition that the spectral radius is an eigenvalue and is equal to one. (For future reference we observe that, whenever there is a spectral gap,

$$
\tilde{T}(t) m \rightarrow c \bar{m} \quad \text { as } t \rightarrow \infty
$$

exponentially in the weak*-sense, for any positive initial measure $m$. Here $c=c(m)$ is a positive real number.)

The defining relations (6.1)-(6.2) are not suitable for "finding" steady states. For that purpose, the generation perspective is much more suitable. In particular one can concentrate on newborn individuals and the offspring they are expected to produce, with due attention to the state-at-birth of the offspring. 
In the simple case of one possible state-at-birth, a first steady state condition is that the basic reproduction ratio, the expected number of offspring, equals one:

$$
R_{0}(\bar{I}):=L_{\rho(\infty) \bar{I}}\left(x_{b}\right)=1
$$

This is a condition on $\bar{I}$. If $\operatorname{dim} I=1$ this is one equation in one unknown. Very often $R_{0}$ is monotone in $\bar{I}$ which then immediately yields uniqueness.

More generally we should, in the notation of (4.6), have

$$
\Lambda_{\rho(\infty) \bar{I}} \times b=b
$$

with $b$ a positive measure on the set $\Omega_{b}$ of possible birth states. Written out in detail (6.4) reads

$$
\int_{\Omega_{b}} \Lambda_{\rho(\infty) \bar{I}}(x, \omega) b(d x)=b(\omega)
$$

for all measurable subsets $\omega$ of $\Omega_{b}$. And if $\Omega_{b}$ is a nice subset of $\mathbf{R}^{k}$ for some $k$ and $b$ has a density $f$ we may rewrite this as

$$
\int_{\Omega_{b}} \xi L_{\rho(\infty) \bar{I}}(x) f(x) d x=f(\xi), \quad \xi \in \Omega_{b} .
$$

Equation (6.4) is a linear eigenvalue problem: the dominant eigenvalue of a positive operator should be one. This is, just as (6.3) but now more implicitly, a condition on the parameter $\bar{I}$. If this condition is satisfied and the eigenvalue is algebraically simple (a sufficient condition being the irreducibility of the positive operator) then the eigenvector $b$ is determined uniquely modulo a positive multiplicative constant, to be denoted by $c$ below.

Returning to the case of a fixed state-at-birth, we note that $(2.10)-(2.12)$ simplify considerably when the input is constant. For given $\bar{I}$ we define $\bar{x}$ and $\overline{\mathcal{F}}$ by

$$
\begin{gathered}
\begin{cases}\frac{d \bar{x}}{d a} & =g(\bar{x}, \bar{I}) \\
\bar{x}(0) & =x_{b}\end{cases} \\
\begin{cases}\frac{d \overline{\mathcal{F}}}{d a} & -\mu(\bar{x}, \bar{I}) \overline{\mathcal{F}} \\
\overline{\mathcal{F}}(0) & =1\end{cases}
\end{gathered}
$$

and next we note that

$$
R_{0}(\bar{I})=\int_{0}^{\infty} \beta(\bar{x}(a), \bar{I}) \overline{\mathcal{F}}(a) d a .
$$


Let $c$ denote the steady $p$-birth rate. Then

$$
\bar{m}(\omega)=c \int_{0}^{\infty} u_{\rho(a) \bar{I}}\left(x_{b}, \omega\right) d a=c \int_{0}^{\infty} \overline{\mathcal{F}}(a) \delta_{\bar{x}(a)}(\omega) d a
$$

and consequently (6.2) can be written as

$$
\bar{I}=c \int_{0}^{\infty} \overline{\mathcal{F}}(a) \gamma(\bar{x}(a)) d a .
$$

Beware that $\overline{\mathcal{F}}$ and $\bar{x}$ depend on $\bar{I}$.

Theorem $6.1 \bar{m}$ is a steady state, i.e., (6.1)-(6.2) hold, iff $\bar{m}$ is given by (6.10), with $\bar{x}$ and $\overline{\mathcal{F}}$ defined by (6.7)-(6.8), where $\bar{I}$ and $c$ are such that (6.3) (with $R_{0}(\bar{I})$ given by (6.9)) and (6.11) hold.

For the proof see Part III. Note that (6.3) and (6.11) are $1+\operatorname{dim} I$ equations in as many unknowns, viz., $c$ and $\bar{I}$. Also note that (6.9) is defined completely in terms of solutions of ODE, since we may supplement (6.7) - (6.8) with

$$
\begin{cases}\frac{d \bar{L}}{d a} & =\beta(\bar{x}, \bar{I}) \overline{\mathcal{F}} \\ L(0) & =0\end{cases}
$$

and put

$$
R_{0}(\bar{I})=\bar{L}(\infty)
$$

Similarly we may write (6.11) as

$$
\bar{I}=c G(\infty)
$$

where $G$ is obtained by solving

$$
\left\{\begin{array}{ll}
\frac{d G}{d a} & =\gamma(\bar{x}) \overline{\mathcal{F}} \\
G(0) & =0
\end{array} .\right.
$$

The main message of Kirkilionis et al. ( 2001) is that one can do a numerical parameter continuation study of steady states of physiologically structured population problems by combining standard ODE solvers with standard continuation algorithms when solving (6.3)-(6.11). 


\section{$7 \quad$ Linearized stability}

Given a steady state, how do we determine whether or not it is stable? Apart from the special situation in which we want to determine the ability of a missing species to invade successfully an existing community (see e.g. Part III, Sections 2.2 and 2.3 where it is explained that the answer can be given in terms of $R_{0}$ ), this is a difficult question. We say that the answer can be found by way of a characteristic equation if it is possible to derive a function $f: \mathbf{C} \rightarrow \mathbf{C}$ such that the steady state is asymptotically stable if all roots of the equation $f(\lambda)=0$ lie in the left half plane while being unstable if at least one root lies in the right half plane. We claim that for physiologically structured population models the answer can indeed be found by way of a characteristic equation and that, moreover, this equation takes the form

$$
\operatorname{det} M(\lambda)=0,
$$

where $M$ is a $\operatorname{dim} I \times \operatorname{dim} I$ matrix. The intuitive explanation is that the semigroup $\tilde{T}(t)=T_{\rho(t) \bar{I}}$ of positive linear operators introduced in the beginning of Section 6 has dominant eigenvalue zero. Accordingly, the stability or instability is completely determined by the feedback loop (and not by the population dynamics per se) and this leads, after linearization, to a transcendental characteristic equation in terms of a matrix of $\operatorname{size} \operatorname{dim} I \times \operatorname{dim} I$ (essentially the $\lambda$ comes in via the Laplace transform of a time kernel; see below).

The proof of this claim is involved and, in fact, some details still have to be filled in. For the stability part there are two steps:

Step 1 assuming that $I_{m}(t)-\bar{I} \rightarrow 0$ exponentially for $t \rightarrow \infty$, show that $S(t, m) \rightarrow \bar{m}$ for $t \rightarrow \infty$,

Step 2 assuming that all roots of (7.1) are in the left half plane, show that $m-\bar{m}$ small implies that $I_{m}(t)-\bar{I} \rightarrow 0$ for $t \rightarrow \infty$ (in fact exponentially).

As usual, the instability part is more difficult (note that, for instance, the instability part was not proved in the book by Webb (1985); it was proved, for age structured models, by Prüß(1983); see also (Desch and Schappacher 1986; Clément et al. 1987). The difficulty is substantially enhanced in the present case by the fact that the nonlinear semigroup is not differentiable (indeed, there is a problem with, e.g., slightly shifted Dirac easures). Our "escape strategy" is to consider an invariant and attracting subset of the $p$ state space on which we have more smoothness. In work in progress, mainly 
by Philipp Getto, we use a different $p$-state representation to characterize this subset, viz., we use the history of $I$ and the history of the population birth rate to identify the $p$-state. In our further description below we restrict our attention to the stability part.

The only nonlinear feature in the constructive definition of the semiflow $S$ is the fixed point problem (5.1) for the environmental variable $I$. So that is the problem we should linearize. As a preparatory step we rewrite (5.1) in the form

$$
I(t)-\bar{I}=\gamma \times\left(T_{\rho(t) I}-T_{\rho(t) \bar{I}}\right) \bar{m}+\gamma \times T_{\rho(t) I}(m-\bar{m})
$$

and introduce the map $Q$ that describes how the output depends on the perturbation of the steady input

$$
(Q J)(t)=\gamma \times\left(T_{\rho(t)(\bar{I}+J)}-T_{\rho(t) \bar{I}}\right) \bar{m} .
$$

Now think of $J$ as extended by zero for negative arguments and define the shift operator $\theta(s)$ by

$$
(\theta(s) J)(\tau)=J(\tau-s)
$$

Proposition $7.1 Q$ is translation invariant:

$$
Q \theta(s)=\theta(s) Q
$$

Even though the map $J \mapsto T_{\rho(t)(\bar{I}+J)} \bar{m}$ is in general not smooth, the map $Q$ may very well be, as it involves the pairing with $\gamma$. We state this as an assumption.

Assumption $7.2 Q$ is differentiable with derivative $L$.

This is basically a smoothness assumption on $\gamma$. Admittedly the assumption is stated rather imprecisely, as we have not specified the function space of inputs. The idea, however, is to compute the derivative for any fixed $t$ and to use the outcome to define a linear input-output map $L$.

Now observe that $L$ inherits the translation invariance of $Q$ and recall that "linear + translation invariant $\Rightarrow$ convolution" whence we have

Proposition $7.3(L J)(t)=\int_{0}^{t} k(t-\tau) J(\tau) d \tau$ for some kernel $k$. 
Finally, we define $M(\lambda)$ to be the Laplace transform of $k$ minus the identity. In fact one can express $k$, and hence $M$, explicitly in terms of solutions of linearized ODE like, when one linearizes (2.10)

$$
\frac{d Y}{d a}=\frac{\partial g}{\partial x} Y+\frac{\partial g}{\partial I} J
$$

We refer to Kirkilionis et al. (2001) for the details. Note that this characterization of $k$ allows a numerical implementation. Thus, despite all the complications, one can make the linearized stability test operational in the context of concrete examples!

\section{References}

Ackleh, A. S. and Ito, K. (to appear). Measure-valued solutions for a hierarchically size-structured population SIAM J. Appl. Math.

Calsina, À. and Saldaña, J. (1997). Asymptotic behaviour of a model of hierarchically structured population dynamics, J. Math. Biol. 35:967-987.

Clément, Ph., Diekmann, O., Gyllenberg, M., Heijmans, H.J.A.M., Thieme, H.R. (1989). Perturbation theory for dual semigroups III. Nonlinear Lipschitz continuous perturbations in the sun reflexive case. In Volterra integrodifferential equations in Banach spaces and applications, Trento 1987, G. Da Prato and M. Iannelli (Eds.), Pitman research Notes in Mathematics Series, 190, pp. 67-89.

Cushing, J.M. (1998). An introduction to structured population dynamics, CBMS-NSF Regional conference series in applied mathematics 71, SIAM, Philadelphia.

Desch and Schappacher (1986). Linearized stability for nonlinear semigroups. In Differential Equations in Banach Spaces (A. Favini and E. Obrecht, Eds.) Spinger Lecture Notes in Mathematics 1223, pp. 61-73.

Diekmann, O. and Getto, Ph. (to appear). Boundedness, global existence and continuous dependence for nonlinear dynamical systems describing physiologically structured populations, Journal of Differential Equations.

Diekmann, O., Gyllenberg, M., Metz, J.A.J., and Thieme, H.R. (1998). On the formulation and analysis of general deterministic structured population models: I Linear theory. Journal of Mathematical Biology 36: 349-388.

Diekmann,O., Gyllenberg, M., Huang, H., Kirkilionis, M., Metz, J.A.J. and Thieme, H.R. (2001). On the Formulation and Analysis of General Deter- 
ministic Structured Population Models. II. Nonlinear Theory. Journal of Mathematical Biology 43: 157-189.

Diekmann, O., Gyllenberg, M. and Metz, J.A.J. (2003). Steady-state analysis of structured population models, Theoretical Population Biology 63:309-338. Getto, Ph., Diekmann, O. and de Roos A.M. (submitted). On the (dis)advantages of cannibalism, submitted to Journal of Mathematical Biology.

Kooijman, S.A.L.M. (2000). Dynamic Energy and Mass Budgets in Biological Systems, Cambridge University Press, Cambridge.

Kirkilionis, M. and Saldaña, J. (in preparation). A height-structured forest model. http://www.iwr.uni-heidelberg.de/sfb/Preprints2001.html

Kirkilionis, M., Diekmann, O., Lisser, B., Nool, M., de Roos, A.M., and Sommeijer, B. (2001). Numerical continuation of equilibria of physiologically structured population models. I. Theory. Mathematical Models and Methods in Applied Sciences 11: 1101 - 1127.

Kirkilionis et al. (2001).

Metz, J.A.J. and Diekmann, O. (1986). The Dynamics of Physiologically Structured Populations. Lecture Notes in Biomathematics 68. Springer, Berlin.

Persson, L., Byström, P., and Wahlström, E. (2000). Cannibalism and competition in Eurasian perch: Population dynamics of an ontogenetic omnivore, Ecology 81:1058-1071.

Persson, L., De Roos, A.M., Claessen, D., Byström, P., Lövgren, J., Sjögren, S., Svanbäck, R., Wahlström, E., and Westman, E. (2003). Gigantic cannibals driving a whole-lake trophic cascade, PNAS 100:4035-4039

Prüß, J. (1983). Stability analysis for equilibria in age-specific population dynamics, Nonl. Anal. TMA 7:1291-1313.

de Roos, A.M., Person, L. and Thieme, H.R. (2003). Emergent Allee effects in top predators feeding on structured prey populations, Proc. R. Soc. Lond. B 270: 611-618.

de Roos, A.M. and Persson, L. (2001). Physiologically structured models from versatile technique to ecological theory, Oikos 94:51-71.

de Roos, A.M. and Persson, L. (2002). Size-dependent life-history traits promote catastrophic collapses of top predators, Proc. Natl. Acad. Sci. USA 99: 12907-12912) 
de Roos, A.M., Persson, L. and McCauley, E. (2003). The influence of sizedependent life history traits on the structure and dynamics of populations and communities. Ecol. Lett. 6: 473-487.

Scheffer, M., Carpenter, S.R., Foley, J.A., Folke, C. and Walker, B. (2001). Catastrophic shifts in ecosystems, Nature 413: 591-596.

Tucker and Zimmermann (1988). A nonlinear model of population dynamics containing an arbitrary number of continuous structure variables, SIAM J. Appl. Math. 48: 549-591.

Webb, G.F. (1985) Nonlinear Age-Dependent Population Dynamics, Marcel Dekker, New York. 